\title{
Les Nematodirinae (Nematoda) chez les Ruminants et chez les Lagomorphes
}

\author{
par M.-C. DURETTE-DESSET \\ Laboratoire de Zoologie (Vers) associé au C.N.R.S., Muséum national \\ d'Histoire naturelle, 43, rue Cuvier, F 75231 Paris Cedex 05.
}

\section{Résumé.}

Etude de 8 espèces de Nematodirinae et, plus particulièrement, de leur synlophe : Nematodirus filicollis, $N$. spathiger, $N$. helvetianus, $N$. battus, $N$. tortuosus, Nematodirella dromedarii, Nematodiroides zembrae, et Rauschia triangularis, espèce type du nouveau genre Rauschia.

En utilisant les données bibliographiques, nous connaissons les synlophes de 4 autres espèces.

Le synlophe conserve, dans tous les cas, la symétrie bilatérale primitive des Molineidae ; il n'y a, chez les parasites de Ruminants (et ceux de Rongeurs), ni gradient de taille bien marqué, ni orientation privilégiée de la pointe des arêtes, bien nette. Ces signes de spécialisation s'observent au contraire de façon constante chez les parasites de Lagomorphes. Chez ces derniers, il apparaît, à partir du synlophe de type "Anoplostrongylinae ", des tentatives évolutives variées. La plus remarquable réside dans l'hypertrophie des arêtes dorsales, et aboutit, en fin d'évolution, à un enroulement dextre du corps selon la ligne dorsale, qui s'oppose à tout ce que l'on connaît dans la superfamille.

Le nouveau genre Rauschia (espèce type $R$. triangularis) est créé pour les parasites de Lagomorphes précédemment classés dans le genre Nematodirus, car ils ont un synlophe complexe, différent de celui des parasites de Ruminants.

Un tableau dichotomique des 6 genres de Nematodirinae est proposé.

\section{Summary.}

\section{Nematodirinae (Nematoda) from Ruminants and from Lagomorpha.}

Study of eight species of Nematodirinae with special emphasis on their synlophe : Nematodirus filicollis (Rudolphi, 1802), N. spathiger (Railliet, 1896), N. helvetianus May, 1920, 
N. battus Crofton \& Thomas, 1951, N. tortuosus Tucker, 1942, Nematodirella dromedarii (May, 1920), Nematodiroides zembrae (Bernard, 1965) and Rauschia triangularis, type species of the new genus Rauschia.

Furthermore, bibliographical data permit to know the structure of the synlophe in four other species.

In each of these species the synlophe retains the primitive bilateral symmetry observed in the Molineidae ; in species parasitic in Ruminants and Rodents, the synlophe shows non pronounced size gradient, nor a pronounced peculiar orientation of the tip of the crests. These last specialized characters are observed, on the contrary, in species parasitic in Lagomorpha. These latter show, starting from synlophes of the "Anoplostrongylinae »-type, various evolutionary essays ; the most remarkable is an hypertrophy of the dorsal crests which leads at the end of the evolution, to a dextral coiling, the back of the animal being inside the spire : such a position appears unique in the superfamily.

Rauschia gen. nov. (type species: $R$. triangularis) is created for species previously pertaining to Nematodirus parasite of Lagomorpha, and in which the synlophe, very complex, differs from the synlophe of the parasite of Ruminants.

A dichotomic key of the six genera of Nematodirinae is proposed.

\section{Introduction}

Les Nématodes Trichostrongyloidea parasites de Ruminants appartiennent presque tous à la famille des Trichostrongylidae, famille dans laquelle le synlophe est absent ou reste rudimentaire. La seule exception importante est celle des Nématodirinae, qui nous paraissent appartenir à la famille des Molineidae. Il s'agit d'une famille primitive, chez laquelle le synlophe conserve la symétrie bilatérale fondamentale, mais l'étude détaillée de celui-ci montre que, chez des formes apparemment proches les unes des autres et classées jusqu'à maintenant dans le même genre Nematodirus, le synlophe reste simple chez les parasites de Ruminants, alors qu'il est complexe chez les parasites de Lagomorphes.

Tout se passe comme si le tube digestif relativement peu volumineux des Lagomorphes rendait nécessaire un appareil de fixation (synlophe) perfectionné des Nématodes parasites, appareil dont pourraient être démunis les parasites vivant dans le volumineux tube digestif des grands Ruminants.

\section{Première partie : Données morphologiques}

\section{I. - Nematodirus spp., parasites de Ruminants en Europe occidentale.}

Nous disposons, dans nos collections, de quatre espèces.

a. Origine du matériel.

- N. filicollis (Rudolphi, 1802) - (MNHN : 355 CA-356 CA) - Ovis aries Sud-Est de l'Angleterre.

- N. spathiger (Railliet, 1896) (MNHN : 357 CA-358 CA) - Ovis aries - SudEst de l'Angleterre. 
Tableau I. Caractères différentiels de Nematodirus spp. de Ruminants.

\begin{tabular}{|c|c|c|c|c|}
\hline & filicollis & spathiger & helvetianus & battus \\
\hline $\begin{array}{l}\text { corona } \\
\text { radiata }\end{array}$ & 30 denticules & 50 denticules & 60 denticules & 35 denticules \\
\hline $\begin{array}{l}\text { Nombre de } \\
\text { crêtes } \\
\mathrm{v}=\text { ventrales } \\
\mathrm{d}=\text { dorsales }\end{array}$ & $\begin{array}{l}7 \mathrm{v}, \quad 7 \mathrm{~d} \\
7 \mathrm{v}, \quad 7 \mathrm{~d}\end{array}$ & $\begin{array}{l}9 \mathrm{v}, \quad 9 \mathrm{~d} \\
9 \mathrm{v}, \quad 9 \mathrm{~d}\end{array}$ & $\begin{array}{l}15 \mathrm{v}, \quad 15 \mathrm{~d} \\
16 \mathrm{v}, \quad 17 \mathrm{~d}\end{array}$ & $\begin{array}{l}9 \mathrm{v}, 9 \mathrm{~d} \\
10 \mathrm{v}, 10 \mathrm{~d}\end{array}$ \\
\hline $\begin{array}{l}\text { Gradient de } \\
\text { taille des } \\
\text { crêtes }\end{array}$ & $\begin{array}{l}\text { fort gradient } \\
\text { médio-latéral }\end{array}$ & $\begin{array}{c}\text { gradient } \\
\text { méaio-latéral }\end{array}$ & $\begin{array}{l}\text { absence de } \\
\text { gradient }\end{array}$ & $\begin{array}{l}\text { léger gradient } \\
\text { médio-latéral }\end{array}$ \\
\hline Lobe dorsal & $\begin{array}{l}\text { non divisé } \\
\text { en } 2 \text { lobules }\end{array}$ & $\begin{array}{l}\text { divisé en } 2^{\circ} \\
\text { lobules marqués }\end{array}$ & $\begin{array}{l}\text { divisé en } 2 \\
\text { lobules marqués }\end{array}$ & $\begin{array}{l}\text { divisé en } \\
2 \text { lobules } \\
\text { peu marqués }\end{array}$ \\
\hline Rameaux de la 9 & fins & êpais & très épais & moyens \\
\hline $\begin{array}{l}\text { Longueur des } \\
\text { côtes } 2 \text { à } 6\end{array}$ & équivalente & inégale & inêgale & êquivalente \\
\hline Côtes 5 et 6 & $\begin{array}{l}\text { légèrement } \\
\text { divergentes à } \\
\text { leur extrêmité }\end{array}$ & $\begin{array}{l}\quad \text { non } \\
\text { divergentes à } \\
\text { leur extrémité }\end{array}$ & $\begin{array}{l}\quad \text { non } \\
\text { divergentes à } \\
\text { leur extrémité }\end{array}$ & $\begin{array}{l}\text { très } \\
\text { divergentes à } \\
\text { leur extrémité }\end{array}$ \\
\hline $\begin{array}{l}\text { Longueur pointe } \\
\text { des spicules }\end{array}$ & $16 \mathrm{Lm}$ & $16 \mathrm{Lm}$ & $35 \mathrm{Nm}$ & $15 \mathrm{um}$ \\
\hline $\begin{array}{l}\text { Pointe des } \\
\text { spicules }\end{array}$ & allongée & spatulée & très allongée & en cuiller \\
\hline Queue ? & $\begin{array}{l}\text { arrondie avec } \\
\text { pointe caudale }\end{array}$ & $\begin{array}{l}\text { arrondie avec } \\
\text { pointe caudale }\end{array}$ & $\begin{array}{l}\text { arrondie avec } \\
\text { pointe caudale }\end{array}$ & pointue \\
\hline
\end{tabular}



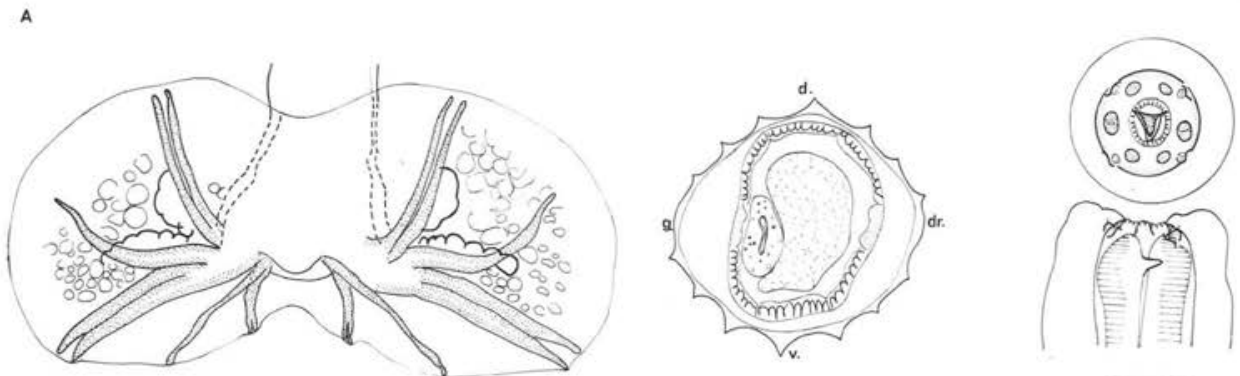

B
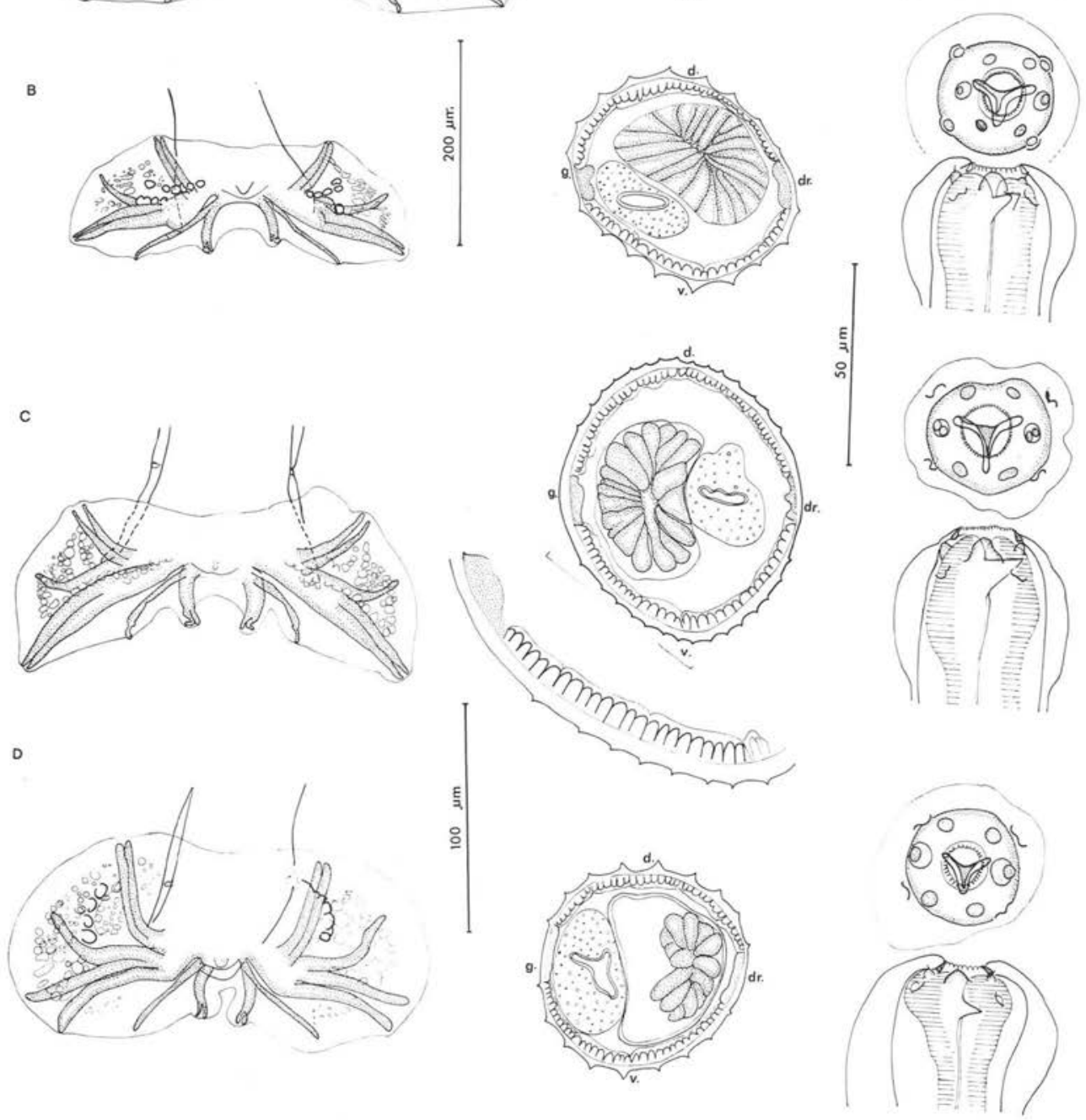

Fig. 1. Nematodirus spp. de Ruminants

A. N. filicollis (Rudolphi, 1802) ; B. N. spathiger (Railliet, 1896) ; C. N. helvetianus May, 1920 ; D. N. battus Crofton et Thomas, 1951.

Pour chaque espèce, nous figurons de gauche à droite la bourse caudale, le synlophe au milieu du corps, la tête en vue apicale et latérale droite.

Bourses caudales : éch. $200 \mu \mathrm{m}$; synlophes : éch. $100 \mu \mathrm{m}$; têtes : éch. $50 \mu \mathrm{m}$. 

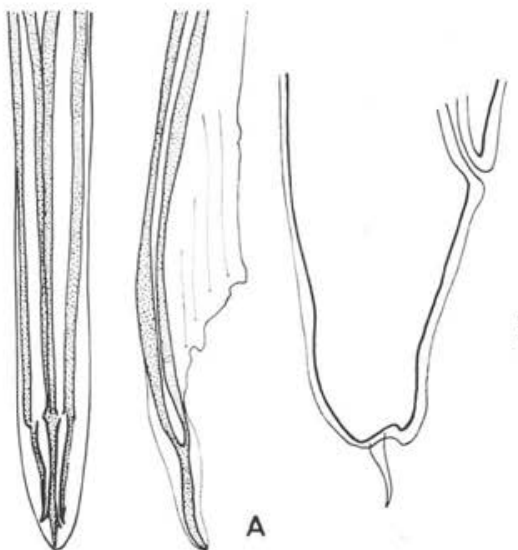

A

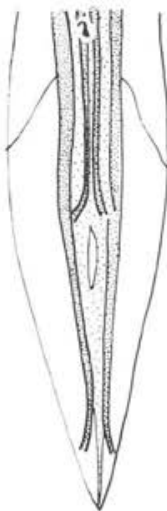

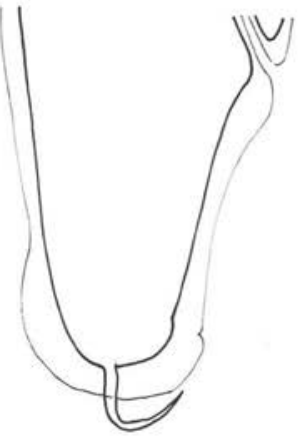

$\mathrm{C}$
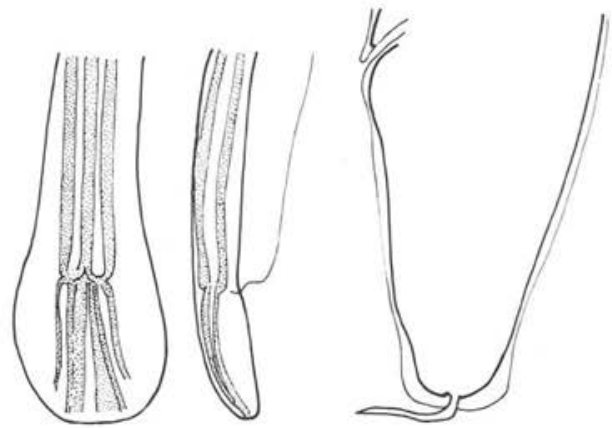

B
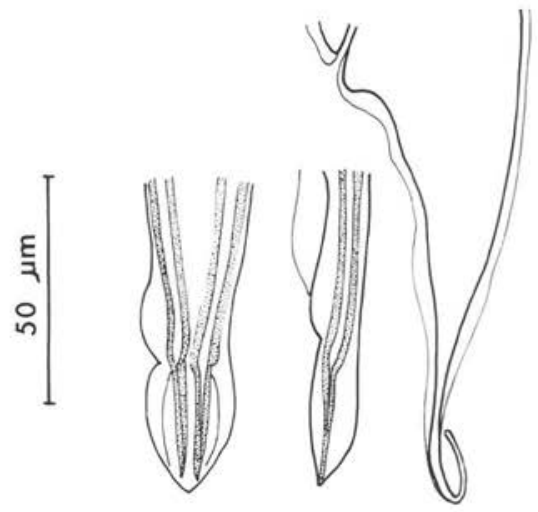

D

Fig. 2. Nematodirus spp. de Ruminants

A. N. filicollis ; B. N. spathiger; C. N. helvetianus; D. N. battus.

Pour chaque espèce, nous figurons de gauche à droite, la pointe des spicules en vue ventrale puis latérale et la queue de la femelle.

Spicules: éch. $25 \mu \mathrm{m}$; queues femelles : éch. $50 \mu \mathrm{m}$.

- N. helvetianus May, $1920-($ MNHN : 970 HA-972 HA) - Bos taurus - Italie. (MNHN : $42 \mathrm{HB})$ - Ovis aries - Haute-Vienne, France.

- N. battus Crofton et Thomas, $1951-(\mathrm{MNHN}$ : 353 CA-354 CA) - Ovis aries

- Sud-Est de 1'Angleterre. 
b. Diffèrenciation des espèces.

Ces espèces sont différenciables par les caractères morphologiques cités dans le tableau I et figurés en 1 et 2 . Les conclusions sont en accord avec celles de J. H. Rose, 1959.

\section{II. - Nematodirus spathiger, parasite de Ctenodactylus gundi.}

Origine du matériel : (MNHN : 736 SB - 737 SB) - Algérie. - (MNHN : 18 HB) -Tunisie.

Nous avons pu également examiner un matériel récolté par Seurat (1916) en Tunisie et étiqueté "Nematodirus filicollis ».

Tous les spécimens (fig. $3, A, B$ ) sont identiques au $N$. spathiger des Ruminants.

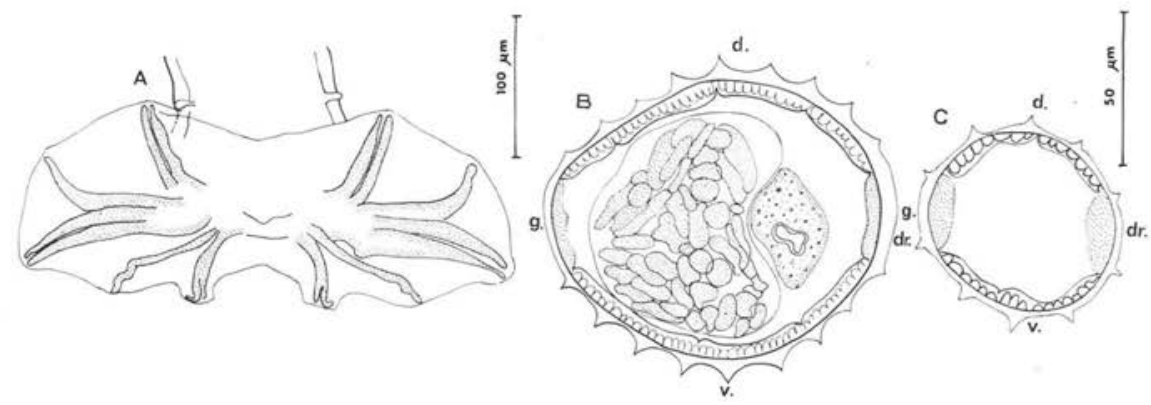

Fig. 3. Nematodirus spathiger

Chez le Gundi : A, bourse caudale, vue ventrale; B, coupe transversale au milieu du corps. Chez Ovis aries: C, coupe transversale du corps d'un quatrième stade larvaire.

A, éch. $100 \mu \mathrm{m} ; \mathrm{B}, \mathrm{C}$, éch. $50 \mu \mathrm{m}$.

III. - Nematodirus tortuosus Tucker, 1942, parasite de l'intestin grêle de Cricétidés.

a. Origine du matériel : (MNHN : $942 \mathrm{HA})$ - Neotoma cinerea - Batch Lake, South End of Jordan lava flow, Malheur County, Oregon, U.S.A. 11-7-1974. - (MNHN : $59 \mathrm{HB})$. - N. cinerea - Bear Creek, Starkey Experimental Forest, Union County, Oregon, U.S.A. 11-9-1975. - $(\mathrm{MNHN}: 58 \mathrm{HB})-N$. lepida - Succor Creek State Park, Malheur County, Oregon, U.S.A., 14-5-1975.

b. Compléments morphologiques.

- Tête (fig. 4, A, B) : Corona radiata composée d'environ 40 denticules.

- Synlophe (fig. 4, C, D). Les crêtes débutent en arrière de la vésicule céphalique et disparaissent à environ $1 \mathrm{~mm}$ en avant de la bourse caudale chez le ${ }^{*}$, et à $400 \mu \mathrm{m}$ en avant de la vulve chez la ๆ. Le nombre des crêtes augmente progressivement d'avant en arrière (40 à $56 \mathrm{chez} \mathrm{le} \sigma^{\star}, 52$ à $61 \mathrm{chez}$ la + ), tandis que leur taille diminue. Absence de gradient de taille. 


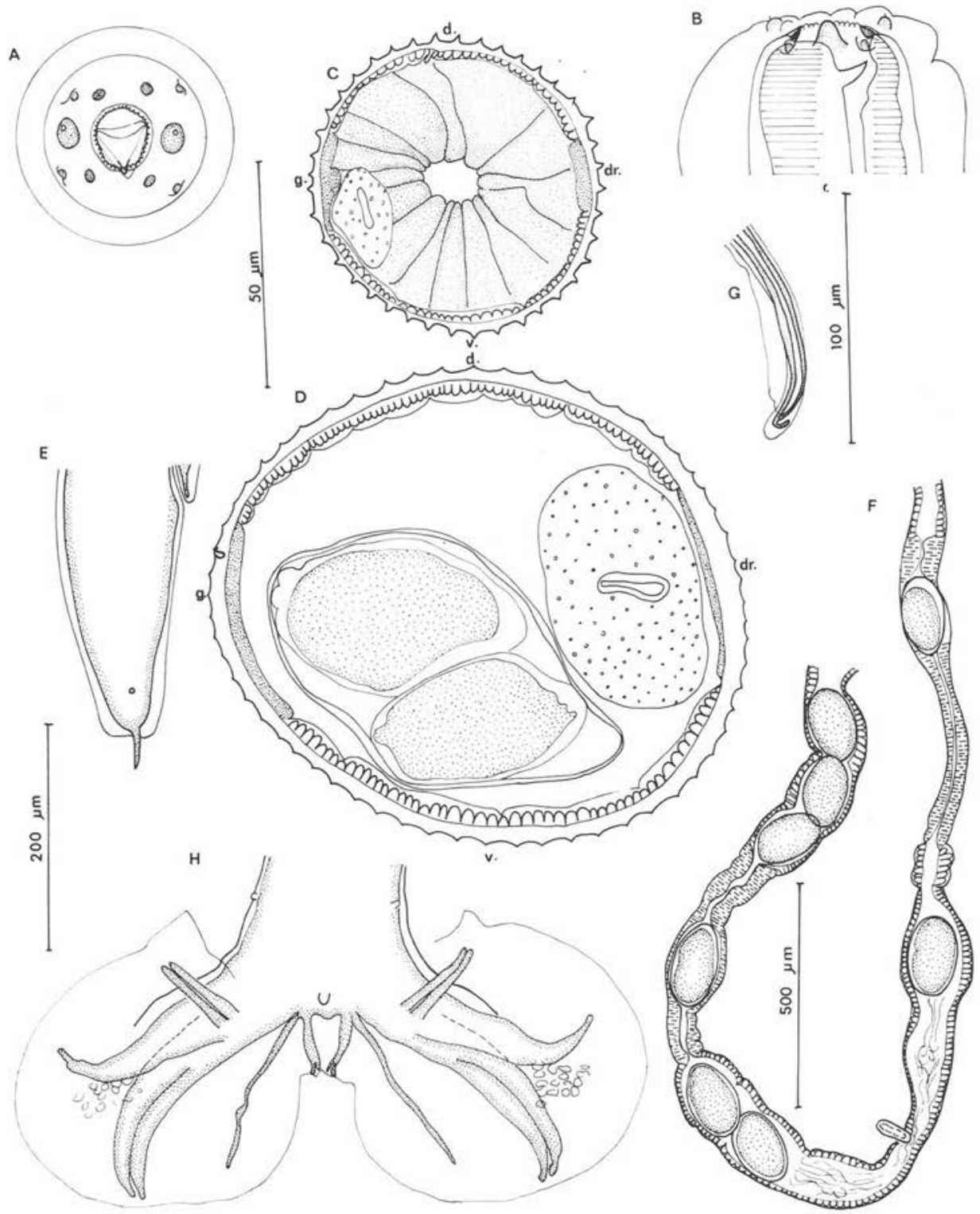

Fig. 4. Nematodirus tortuosus Tucker, 1942

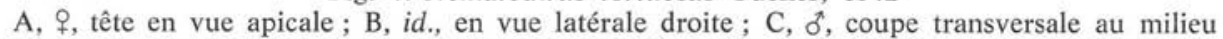

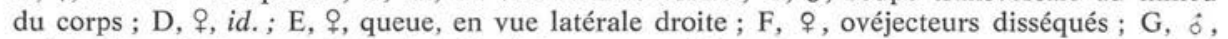
pointe des spicules, vue latérale; $\mathrm{H}, \delta$, bourse caudale, vue ventrale.

A, B, éch. $50 \mu \mathrm{m}$; C, D, E, G, éch. $100 \mu \mathrm{m}$; F, éch. $500 \mu \mathrm{m}$; H, éch. $200 \mu \mathrm{m}$. 
- Mâle (fig. 4, G, H). Spicules ailés, longs de 3,3 mm chez un mâle de 10,5 mm.

- Femelle (fig. $4, F$ ). Vulve à $7,8 \mathrm{~mm}$ de la pointe caudale chez une femelle longue de $21,6 \mathrm{~mm}$. Branche génitale antérieure: vestibule, $550 \mu \mathrm{m}$, sphincter, $100 \mu \mathrm{m}$, trompe, $700 \mu \mathrm{m}$, utérus, $6 \mathrm{~mm}$, contenant 27 œufs hauts de $160 \mu \mathrm{m}$ sur $110 \mu \mathrm{m}$ de large. Branche génitale postérieure : vestibule, $500 \mu \mathrm{m}$, sphincter, $100 \mu \mathrm{m}$, trompe, $500 \mu \mathrm{m}$, utérus, $6,6 \mathrm{~mm}$, contenant 17 œufs. Queue longue de $110 \mu \mathrm{m}$, avec une pointe caudale de $15 \mu \mathrm{m}$.

IV. - Nematodirella dromedarii (May, 1920) Douvres et Lucker, 1958, parasite d'un «Camel».

a. Origine du matériel (MNHN : $969 \mathrm{CA})$ - Bikaner, Indes.

b. Compléments morphologiques.

- Tête (fig. 5, A, B) : Corona radiata composée d'environ 57 denticules.

- Synlophe ( $f i g .5, F$ ) : Les crêtes débutent en arrière de la vésicule céphalique, sont bien marquées dans la partie antérieure, puis disparaissent progressivement. Le tiers postérieur est dépourvu de crêtes. 23 crêtes chez le mâle dans la partie moyenne. Absence de gradient de taille.

- Mâle (fig. 5, E, G, H) : Spicules ailés longs de $5 \mathrm{~mm}$ chez un mâle de 19,4 mm. Pointe commune aux deux spicules longue de $33 \mu \mathrm{m}$.

- Femelle (fig. 5, C, D) : Vulve à $13,6 \mathrm{~mm}$ de la pointe caudale chez une femelle longue de 27,2 mm. Branche génitale antérieure atrophiée. Branche génitale postérieure : vestibule, $1,9 \mathrm{~mm}$, sphincter, $80 \mu \mathrm{m}$, trompe, $350 \mu \mathrm{m}$, utérus, $5,5 \mathrm{~mm}$, avec de nombreux œufs hauts de $200 \mu \mathrm{m}$ sur $100 \mu \mathrm{m}$ de large. Queue longue de $80 \mu \mathrm{m}$, avec une pointe caudale (non mesurée, car cassée).

\section{V. - Nematodirus triangularis Boughton, 1932, parasite de Lepus americanus.}

a. Origine du matériel : (MNHN : $1040 \mathrm{Ka})$ - 5,5 miles. N.E. Blue River, Lane Co., Oregon, U.S.A. - (MNHN : 65 HB) - Tin Trough Pit, Starkey Experimental Forest, Union County, Oregon, U.S.A., 16-9-1975.

b. Compléments morphologiques.

- Enroulement : Généralement dextre (36 individus sur 46) le long de la ligne dorsale dans la moitié antérieure du corps.

- Tête (fig. 6, C, D) : Corona radiata composée d'environ 45 denticules.

- Synlophe (fig. 6, G, $H, I$ ) : Les crêtes débutent derrière la vésicule céphalique et à différents niveaux sur les lignes médianes. Elles disparaissent à environ $500 \mu \mathrm{m}$ en avant de la bourse caudale chez le mâle et au niveau de la queue chez la femelle. 14 crêtes cuticulaires au milieu du corps. Gradient de taille latéro-médian.

- Mâle (fig. 6, $J, K, L$ ) : Spicules ailés, longs de $1,35 \mathrm{~mm}$ chez un mâle de $8,6 \mathrm{~mm}$. Pointe commune aux deux spicules, longue de $25 \mu \mathrm{m}$. 


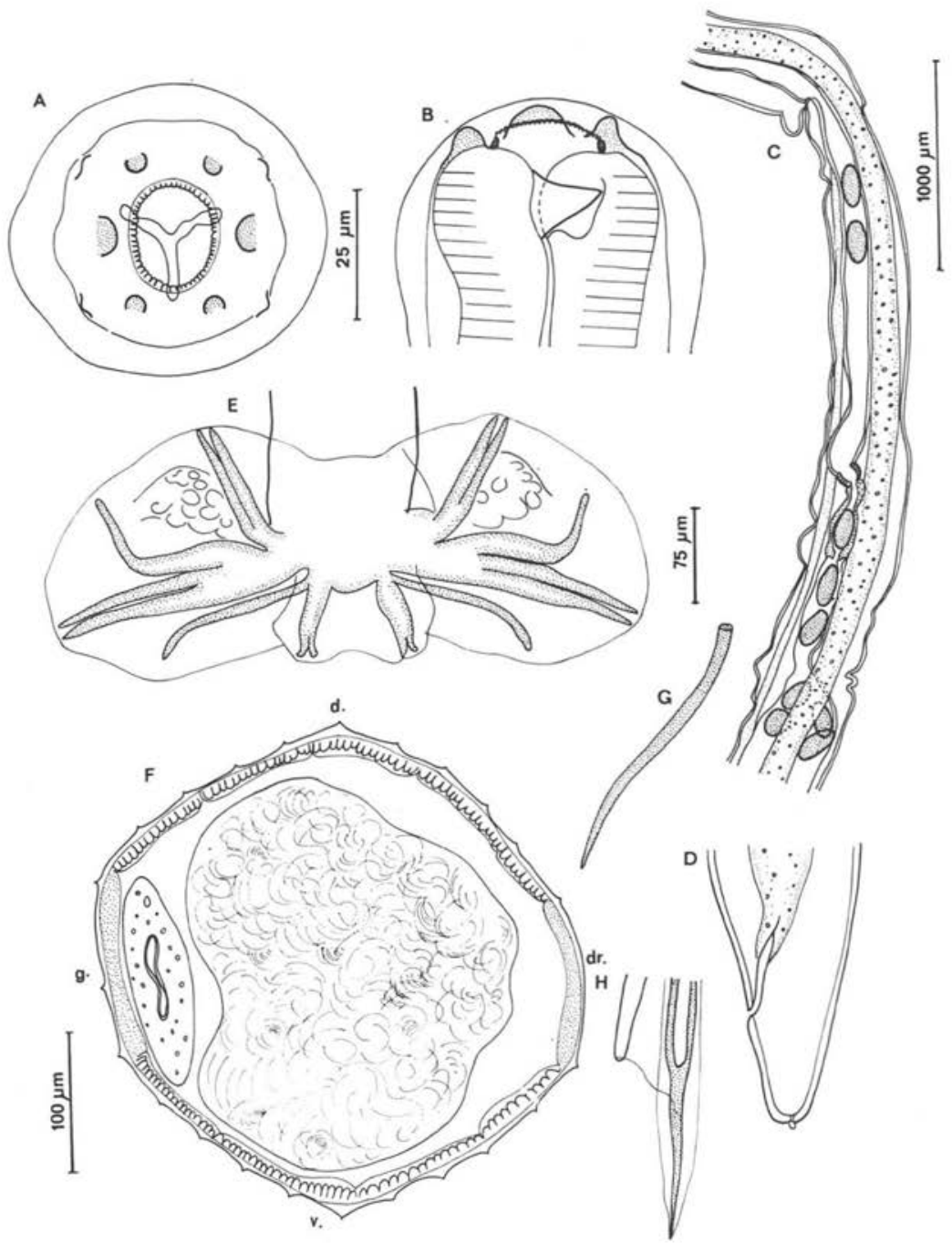

Fig. 5. Nematodirella dromedarii (May, 1920)

A, $\delta^{*}$, tête en vue apicale ; B, id., en vue latérale droite; C, + , région de la vulve et ovéjecteur postérieur; $\mathrm{D}, \uparrow$, vue latérale gauche ; E, $\delta^{*}$, bourse caudale, vue ventrale; $\mathrm{F}, \delta^{*}$, coupe transversale au milieu du corps; $\mathrm{G}, \delta^{\star}$, spermatozoïde; $\mathrm{H}, \delta^{\star}$, pointe des spicules, vue latérale.

A, B, G, H, éch. $25 \mu \mathrm{m}$; C, éch. $1000 \mu \mathrm{m}$; D, E, éch. $75 \mu \mathrm{m}$; F, éch. $100 \mu \mathrm{m}$. 


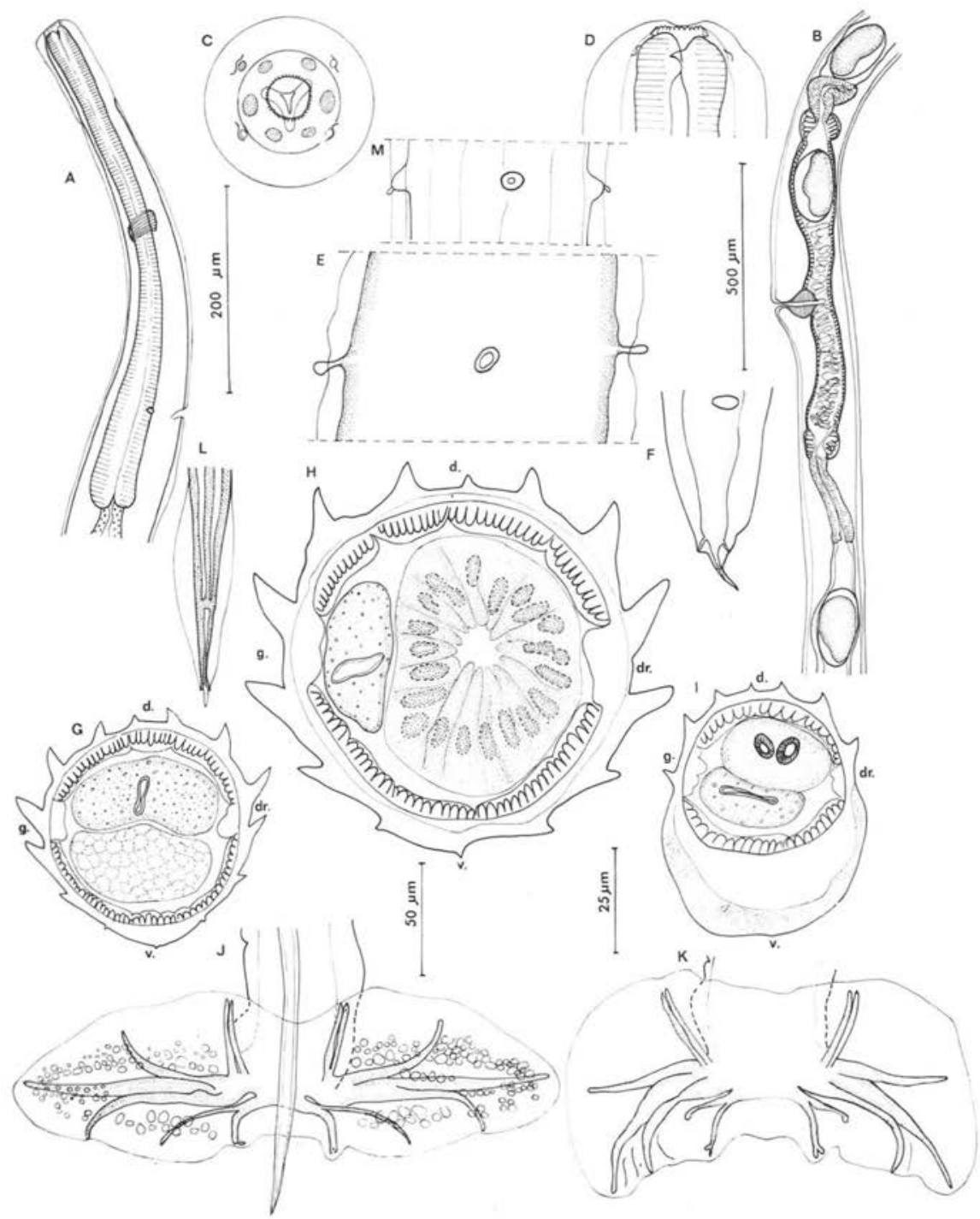

Fig. 6. Rauschia triangularis (Boughton, 1932) n. cb.

A, + , extrémité antérieure, vue latérale droite ; $\mathrm{B}$, + , région des ovéjecteurs, vue latérale gauche; C, D, + , tête successivement en vue apicale et latérale gauche; E, \&, région du pore excréteur et des deirides, vue ventrale; F, $q$, queue, vue ventrale; $\mathrm{G}, \mathcal{q}$, coupe transversale dans le tiers antérieur du corps; $\mathrm{H}, \mathrm{I}, \delta^{*}$, coupes transversales successivement au milieu du corps et dans le tiers postérieur ; J, §o, bourse caudale bien étalée, vue ventrale; K, autre $\delta$, bourse caudale mal étalée, vue ventrale; $\mathrm{L}, \widehat{\delta}$, pointe des spicules. - Nematodirus filicollis. $\mathbf{M}, \delta^{\star}$, région du pore excréteur et des deirides, vue ventrale.

A, J, K, éch. $200 \mu \mathrm{m}$; C, D, E, H, L, M, éch. $25 \mu \mathrm{m}$; B, éch. $500 \mu \mathrm{m}$; F, G, I, éch. $50 \mu \mathrm{m}$. 
- Femelle (fig. 6, $A, B, F$ ) : Vulve à $3 \mathrm{~mm}$ de la pointe caudale chez une femelle longue de 10,3 mm ; longueur de sphincter à sphincter, $830 \mu \mathrm{m}$. Branche utérine antérieure, $800 \mu \mathrm{m}$, avec 7 œufs hauts de $170 \mu \mathrm{m}$ sur $95 \mu \mathrm{m}$ de large. Branche utérine postérieure, $1500 \mu \mathrm{m}$, avec 7 œufs. Queue longue de $85 \mu \mathrm{m}$, pointe caudale, $10 \mu \mathrm{m}$.

c. Remarque.

Dikmans, 1937, différencie N. arizonensis (parasite de Lepus alleni aux U.S.A.) de $N$. triangularis par deux caractères: la longueur comprise entre les 2 sphincters $(800 \mu \mathrm{m}$ à $850 \mu \mathrm{m}$ chez $N$. triangularis, $500 \mu \mathrm{m}$ à $520 \mu \mathrm{m}$ chez $N$. arizonensis $)$ et la position de la vulve (3,6 à $4 \mathrm{~mm}$ chez triangularis, 2,0 à $2,2 \mathrm{~mm}$ chez arizonensis). Chez les 26 femelles étudiées, la longueur comprise entre les 2 sphincters variait de $595 \mu \mathrm{m}$ à $1080 \mu \mathrm{m}$, et la position de la vulve de $2,15 \mathrm{~mm}$ à $3,17 \mathrm{~mm}$.

Nos spécimens nous paraissent donc avoir des mensurations intermédiaires entre les deux espèces. Comme tous les autres caractères sont identiques; il est possible que $N$. arizonensis soit synonyme de $N$. triangularis.

VI. - Nematodiroides zembrae (Bernard, 1965), Bernard, 1967, parasite d'Oryctolagus cuniculus.

a. Origine du matériel (MNHN : $16 \mathrm{HB}, 17 \mathrm{HB})$ - Mendigorria, province de Navarre, Espagne.

b. Compléments morphologiques.

- Enroulement : Généralement dextre (11 individus sur 13), le long de la ligne ventrale sur tout le corps chez le mâle, et dans la partie antérieure chez la femelle.

- Tête (fig. 7, A, B) : Corona radiata composée d'environ 37 denticules.

- Synlophe (fig. 7, $G$ ) : Les crêtes débutent derrière la vésicule céphalique et disparaissent à environ $800 \mu \mathrm{m}$ en avant de la bourse caudale chez le mâle et $500 \mu \mathrm{m}$ en avant de la vulve chez la femelle; 18 crêtes au milieu du corps. Gradient de taille ventro-dorsal.

- Mâle (fig. 7, $H, I$ ) : Spicules longs de $4 \mathrm{~mm}$ chez un mâle de 10,4 mm.

- Femelle (fig. 7, $C, D, E, F$ ) : Vulve à $9,5 \mathrm{~mm}$ de la pointe caudale chez une femelle longue de 14,6 mm. Branche génitale antérieure atrophiée, longue de $620 \mu \mathrm{m}$. Branche génitale postérieure: Vestibule divisé en 2 parties, la première muscularisée est longue de $700 \mu \mathrm{m}$, la seconde de $1,2 \mathrm{~mm}$. Sphincter très allongé, long de $400 \mu \mathrm{m}$. Trompe assez courte : $550 \mu \mathrm{m}$. Queue longue de $80 \mu \mathrm{m}$, pointe caudale, $18 \mu \mathrm{m}$.

\section{Deuxième partie: Évolution du synlophe et classification des Nematodirinae}

Nous connaissons actuellement le synlophe des 8 espèces traitées plus haut, ainsi que celui des espèces suivantes décrites ou redécrites récemment (1): Nematodirus davtianus alpinus Biocca et coll., 1974, Rauschia aspinosus (Schulz, 1931), qui sera

(1) May, 1920, donne d'excellents dessins du synlophe de N. spathiger. 


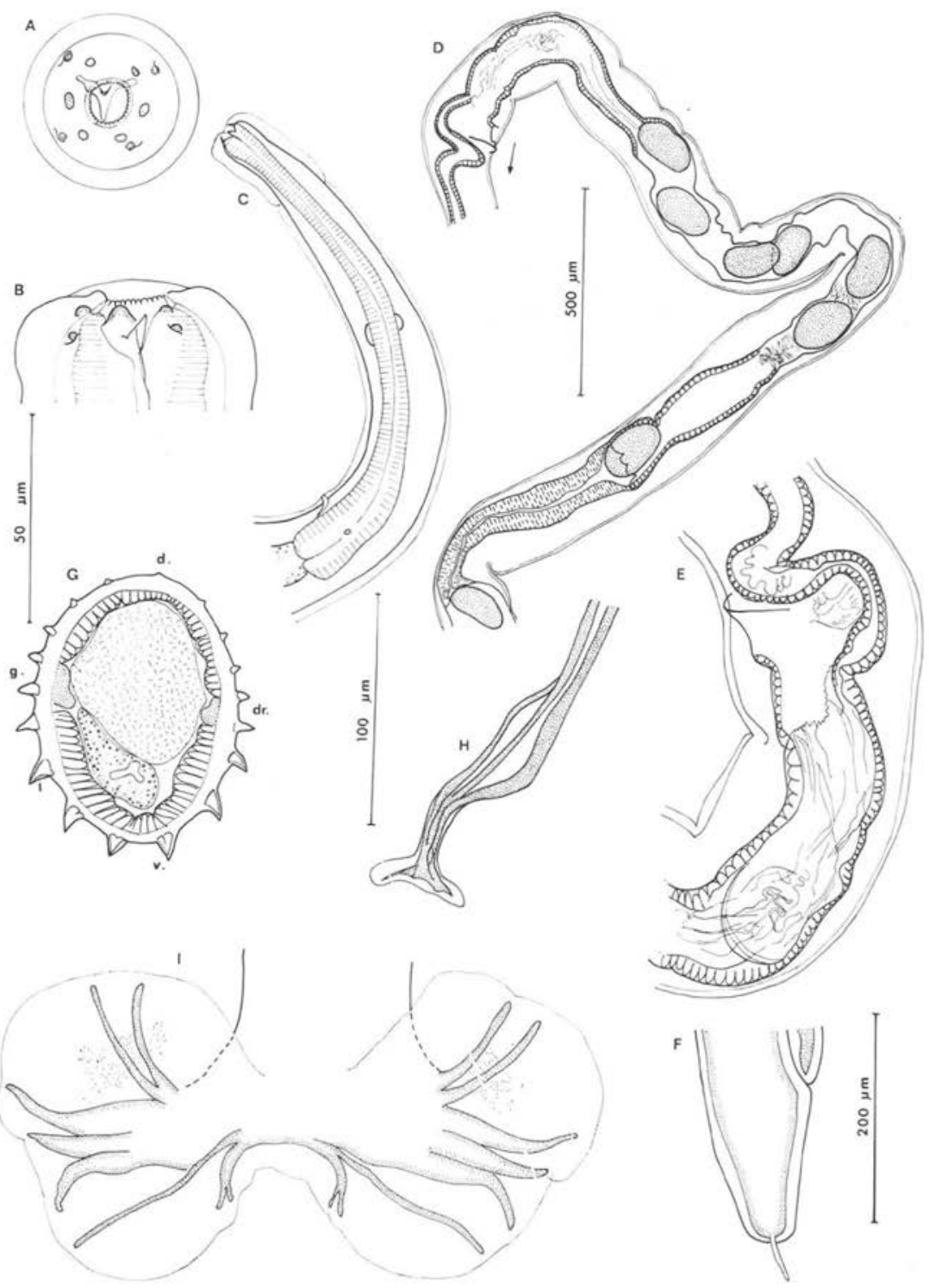

Fig. 7. Nematodiroides zembrae (Bernard, 1965)

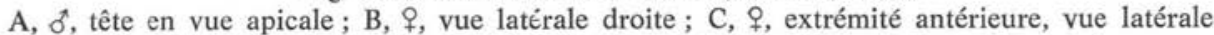
gauche ; D,, , ovéjecteur postérieur, vue latérale gauche ; la flèche indique l'avant du corps ; $\mathrm{E}, \mathfrak{+}$, détail de la vulve, du vagina vera et du début des deux ovéjecteurs; $\mathrm{F}, \mathfrak{q}$, queue, vue laterale droite; $\mathrm{G}, \delta$, coupe transversale au milieu du corps; $\mathrm{H}, \delta$, pointe d'un spicule; I, $\delta$, bourse caudale, vue ventrale.

A, B, éch. $50 \mu \mathrm{m}$; C, E, I, éch. $200 \mu \mathrm{m}$; D, éch. $500 \mu \mathrm{m}$; F, G, H, éch. $100 \mu \mathrm{m}$. 
redécrit ultérieurement, Murielus harpespiculus Dikmans, 1935 (cf. Durette-Desset, 1974) et Murielus abei Durette-Desset, 1974.

L'élément caractéristique du synlophe de la famille des Molineidae réside dans une symétrie bilatérale parfaite. Nous pensions (cf. Durette-Desset et Chabaud, 1977) que la seule élaboration remarquable du synlophe de cette famille était l'orientation dorso-ventrale de la pointe des crêtes, caractéristique des Anoplostrongylinae. Il apparaît aujourd'hui que le synlophe des Nematodirinae est parfois complexe et que, tout en conservant la symétrie bilatérale primitive, de nombreuses tentatives évolutives ont eu lieu dans cette sous-famille, portant soit sur le gradient de taille des crêtes, soit sur l'orientation de leur pointe.

Les douze synlophes que nous connaissons se divisent en 2 groupes : six espèces n'ont pas d'orientation des crêtes [ou une orientation à peine indiquée (N. battus)] Six autres espèces, au contraire, ont une orientation bien nette.

Quel que soit le genre dans lequel les espèces sont actuellement rangées, le premier groupe se rencontre chez les Ruminants, et parfois les Rongeurs, alors que le second groupe est caractéristique des Lagomorphes.

Cette corrélation entre l'hôte et le type du synlophe nous apparaît donc comme un élément important pour comprendre l'évolution de la sous-famille et pour sa systématique.

Le synlophe d'une espèce telle que Nematodirus filicollis, ou N. spathiger, constitué de crêtes peu nombreuses et d'assez grande taille, n'évoque pas un processus d'atrophie progressive des crêtes, comme nous en connaissons des exemples chez les Heligmonellidae (dans ce cas, il y a multiplication des arêtes et diminution de leur taille).

La comparaison entre le synlophe du $4^{\mathrm{c}}$ stade et de l'adulte de $N$. spathiger n'évoque pas non plus un processus d'atrophie en cours d'évolution (cf. fig. 3, $B, C$ ).

D'un point de vue morphologique, nous devons donc considérer le synlophe des parasites de Lagomorphes comme plus spécialisé que celui des Ruminants.

Ce fait est en contradiction avec ce que nous savons sur l'évolution générale des Trichostrongyloides, où toutes les autres lignées parasites de Ruminants ont pour ancêtre des parasites de Lagomorphes.

Nous devons donc admettre une division très précoce des Nematodirinae en 2 lignées distinctes: l'une s'étant diversifiée chez les Ruminants sans évolution du synlophe, l'autre, chez les Lagomorphes, avec, au contraire, une forte diversification de cet appareil.

En ce qui concerne les Rongeurs, Nematodirus tortuosus, parasite d'un Cricétidé, n'a aucun élément morphologique important qui le sépare des parasites de Ruminants. Il s'agit donc d'un phénomène de capture. Il eût été tentant d'imaginer $N$. spathiger comme une forme primitive, puisqu'il parasite également un Rongeur très archaïque : Ctenodactylus gundi, mais, dans des cas comparables, on observe de forts phénomènes de spéciation entre le Nématode parasite de l'hôte ancestral et les Nématodes qui ont colonisé les hôtes plus récents. Nous devons donc considérer $N$. spathiger comme un simple parasite transfuge chez le Gundi. 
L'évolution du synlophe chez les Lagomorphes paraît s'effectuer à partir du type Anoplostrongylinae (sous-famille de Molineidae caractéristique des Xénarthres et des Microchiroptères américains), c'est-à-dire à partir de crêtes sans gradient de taille et orientées du ventre vers le dos. $\mathrm{La}_{4}$ de Rauschia aspinosus appartient à ce type.

L'évolution s'effectue d'une part par l'acquisition de gradients de taille très marqués et de types variés (latéro-ventral chez Nematodiroides, médio-latéral chez $R$. triangularis, latéro-médian chez Rauschia aspinosus), d'autre part par l'apparition d'axes d'orientation différents (latéro-ventral chez Nematodiroides, latéro-médian chez Rauschia aspinosus).

Etant conduite à admettre une séparation précoce en 2 lignées, parasites de Lagomorphes, d'une part, parasites de Ruminants, d'autre part, nous ne pouvons conserver un mélange d'espèces des deux lignées dans le même genre.

Nous proposons donc le nouveau genre Rauschia pour désigner les Nematodirus parasites de Lagomorphes. Deux éléments différentiels accessoires paraissent pouvoir s'ajouter aux caractères fournis par le synlophe. Chez les espèces parasites de Ruminants et de Rongeurs, le synlophe s'arrête, chez la femelle, au niveau de la vulve, et les deirides ont une forme comparable à celle qui est figurée en $6, M$. Chez les espèces parasites de Lagomorphes, le synlophe persiste au-delà de la vulve et les deirides ont une forme comparable à celle qui est figurée en $6, E$.

\section{- Définitions :}

Rauschia n. gen., Nematodirinae avec partie antérieure du corps fortement enroulée, souvent selon une spire dextre. Synlophe pourvu de crêtes cuticulaires se poursuivant, chez la femelle, en arrière de la vulve et possédant un ou deux axes d'orientation privilégiés. Gradients de taille bien marqués. Deirides arrondies sans pointe terminale. Didelphie. Parasites de Lagomorphes holarctiques.

- Espèce type : Rauschia triangularis (Boughton, 1932) n. cb.

$=$ (Nematodirus triangularis Boughton, 1932).

- Autres espèces : - R. aspinosus (Schulz, 1931) n. cb., R. leporis (Chandler, 1924) n. cb., R. neomexicanus (Dikmans, 1937) n. cb., R. petrovi (Ivaschkin, 1954) n. cb.

- Nematodirus Ransom, 1907, Nematodirinae dont le corps est soit đéroulé, soit enroulé de façon lâche toujours selon une spire senestre. Synlophe pourvu de crêtes cuticulaires ne se poursuivant jamais chez la femelle en arrière de la vulve et ne possédant pas d'axe d'orientation privilégié. Gradients de taille nuls ou peu marqués. Deirides arrondies avec pointe terminale. Didelphie. Parasites de Ruminants et de Rongeurs. Cosmopolite.

- Espèce type: Nematodirus filicollis (Rudolphi, 1802) Ransom, 1907.

- Autres espèces : voir la liste donnée par Kulmamatov, 1974. 


\section{- Origine géographique des Nematodirinae :}

La similitude entre les synlophes les plus primitifs de Nematodirinae parasites de Lagomorphes et ceux des Anoplostrongylinae (caractéristiques de Xénarthres et Microchiroptères américains) et l'existence du genre Lamanema (parasite de Ruminants au Pérou), qui nous paraît être un Nematodirinae primitif, nous incite à supposer que la sous-famille s'est individualisée à partir des Molineinae en zone néotropicale. On remarquera, en outre, que les Nematodirinae parasites de Ruminants sont largement répandus dans le monde, mais paraissent absents chez les Ruminants sauvages d'Afrique.

\section{- Clef dichotomique des Nematodirinae :}

1 - (2) Côte dorsale non divisée à sa base.

Parasite de Lama ................. Lamanema Becklund, 1963

2 - (1) Côte dorsale divisée en 2 rameaux à sa base.

3 - (8) Synlophe avec axe d'orientation des crêtes bien marqué.

Parasite de Lagomorphes.

4 - (7) Didelphie, la branche génitale postérieure étant ou non fonctionnelle. Parasite de Leporidae (une espèce signalée aussi chez un Ochotone).

5 - (6) Didelphie avec branche génitale postérieure fonctionnelle. Pointe des crêtes ventrales orientée vers le dos .......... Rauschia $\mathrm{n}$. gen.

6 - (5) Didelphie avec branche génitale postérieure non fonctionnelle. Pointe

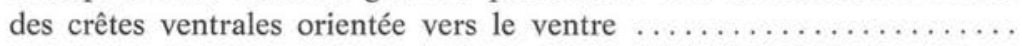
........................ Nematodiroides (Bernard, 1965)

7 - (4) Monodelphie.

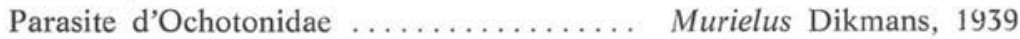

8 - (3) Synlophe sans axe d'orientation ou avec axe d'orientation à peine marqué. Parasite de Ruminants et de Rongeurs.

9 - (10) Didelphie, la branche antérieure étant fonctionnelle

Nematodirus Ransom, 1907

10 - (9) Didelphie, la branche génitale antérieure n'étant pas fonctionnelle ... .................. Nematodirella Yorke et Maplestone, 1926.

\section{Troisième partie :}

\section{Anatomie du synlophe et mode d'enroulement du corps}

Dans tous les cas que nous avons pu observer in vivo chez les Trichostrongyloidea, nous avons constaté que le mode d'enroulement du Ver autour de la villosité se conserve sur le spécimen fixé en extension maximale dans l'alcool chaud. Nous pen- 
sons donc que l'examen de spécimens de collections traduit la position physiologique du Ver tel qu'il est fixé à la muqueuse.

Les Nématodes Trichostrongyloidea qui ont un synlophe complexe appartiennent aux deux grandes familles des Heligmonellidae - Heligmosomidae. Chez les nombreuses espèces que nous connaissons, le corps du Ver est enroulé de façon senestre le long de sa ligne ventrale, si bien que la face du Ver appliquée sur la villosité et s'accrochant à la muqueuse est la face ventrale-gauche (cf. Durette-Desset, 1971). Dans tous les cas, la portion ventrale-gauche du corps, la plus fonctionnelle, est également morphologiquement la plus spécialisée. Lorsqu'il existe une asymétrie quelconque, les arêtes de ce secteur sont plus nombreuses ou plus grandes.

Le cas des Nematodirinae à synlophe complexe est tout à fait particulier, puisque, dans ce cas, nous avons conservation de la symétrie bilatérale primitive.

\section{Nos observations sont les suivantes :}

- Espèces de Ruminants, avec synlophe non orienté et sans gradient de taille:

Corps non enroulé régulièrement, parfois de façon dextre, parfois de façon senestre, mais toujours le long de la ligne ventrale.

- Nematodiroides zembrae, avec arêtes ventrales plus grandes que les dorsales: Corps enroulé le long de la ligne ventrale, généralement de façon dextre, parfois senestre.

- Murielus abei, avec arêtes dorsales légèrement plus grandes que les ventrales: Corps peu enroulé, mais courbé le long de la ligne ventrale, de façon senestre.

- Rauschia triangularis, avec arêtes dorsales nettement plus grandes que les ventrales: Corps ayant un enroulement généralement dextre, parfois senestre, mais, cette fois, enroulé le long de la ligne dorsale.

Cette espèce nous paraît donc très intéressante, puisque c'est le seul Trichostron gyloidea que nous connaissions qui possède ce mode d'enroulement.

Tout se passe comme si une hypertélie aberrante, la croissance des crêtes dorsales, constituait une préadaptation morphologique, finalement utilisée par le Ver, grâce à une inversion du mode d'enroulement, et cela contrairement à la règle suivie par toutes les autres espèces de la superfamille.

\section{REMERCIEMENTS}

Nous remercions très vivement tous les Collègues qui ont récolté le matériel étudié ou nous l'ont transmis : $\mathrm{M}^{\mathrm{mes}} \mathrm{L}$. Gibbons, du Commonwealth Institute of Helminthology (Grande-Bretagne), N. Haffoudhi, de la Faculté de Médecine et de Pharmacie de Tunis, D. Kerbœuf, de l'I.N.R.A., Tours, C. Louzis du Laboratoire Central de Recherches Vétérinaires de Maisons-Alfort; MM. Ben Rachid, de la Faculté de Médecine et de Pharmacie de Tunis, J. Bernard, de la Station Zoologique appliquée de l'Etat, Gambloux (Belgique), Balbo. de l'Universita degli studi di Torino (Italie), G. Jolivet, de l'I.N.R.A., Paris, P. Marchand, de l'Ecole Vétérinaire de Maisons-Alfort, R. L. Rausch, de l'University of Washington, Seattle (U.S.A.), et J.H. Rose, du Central Veterinary Laboratory, Weybridge (Grande-Bretagne). 


\section{Bibliographie}

Becklund W. W. (1963): Lamanema chavezi gen.n., sp.n. and Nematodirus lamae sp.n. (Nema toda: Trichostrongylidae) from the alpaca, Lama pacos, and the vicuna, Vicugna vicugna, in Peru. J. Parasitol, 49, 1023-1027.

Bernard J. (1965): Premières données sur les helminthes parasites du lapin domestique, Oryctolagus cuniculus L. en Tunisie. Arch. Inst. Pasteur Tunis, 42, 193-204.

Bernard J. (1965): Contribution à la connaissance de la faune parasitaire de l'île de Zembra. I. Parasites du lapin, Oryctolagus cuniculus L. Arch. Inst. Pasteur Tunis, 42, 193-204.

Bernard J. (1967): Note complémentaire au sujet de Nematodirus (=Nematodiroides n. gen.) zembrae. Arch. Inst. Pasteur Tunis, 44, 443-447.

Biocca E., Balbo T., Costantini R. (1974): Nematodirus davtiani alpinus subsp. n., Gastrointestinal nematode from Steinbock, Capra ibex, and Chamois, Rupicapra rupicapra, of the parco nazionale del Gran Paradiso, Italian Western Alps. Parassitologia, 16, 57-60.

Boughton R.V. (1932): The influence of helminth parasitism on the abundance of the snowshoe rabbit in Western Canada. Can. J. Res., 7, 524-547.

Chandler A.C. (1924): Some parasitic round worms of the rabbit with descriptions of two new species. Proc. U.S. Natn. Mus., 66, 1-6.

Crofton H. D., Thomas R. J. (1951): A new species of Nematodirus in sheep. Nature, $168,559$.

Dikmans G. (1937): A note on the members of the nematode genus Trichostrongylus occurring in rodents and lagomorphs with descriptions of two new species. J. Wash. Acad. Sci., 27, 203. 209.

Dikmans G. (1939): Two new Nematodes (Trichostrongyloidea) from rodents. Proc. helminth. Soc. Wash., 6, 1-16.

Douvres F. W., Lucker J. T. (1958): A note on the Genera Nematodirus Ransom, 1907 and Nematodirella Yorke and Maplestone, 1926 (Nematoda: Trichostrongylidae), Proc. helminth. Soc. Wash., 25, 48-52.

Durette-Desset M.-C. (1974): Murielus abei n. sp. (Nematoda, Heligmosomidae) parasite d'un Lagomorphe du Népal, et remarques sur le genre Murielus, Dikmans, 1939. Bull. Mus. Nat. Hist. Nat., $3^{e}$ sér., $\mathrm{n}^{\circ}$ 216, Zool. 144, 419-424.

Durette-Desset M.-C., Chabaud A.-G. (1977): Essai de classification des Nématodes Trichostrongyloidea. Ann. Parasitol. Hum. Comp., 52, 539-558.

Ivashkin V.M. (1954): (Helminths of hares in Mongolia). Trudi Gelmintologicheskoi Laboratorii Akademiya Nauk SSSR, 7, 220-225.

Kulmamatov A. (1974): The species composition of the genus Nematodirus Ransom 1907. Mater. Nauchnykh Konferentsii vsesouyznogo Obschestva gel'mintologii, 26, 137-140.

May H.G. (1920): Observations on the nematode genus Nematodirus with descriptions of new species. Proc. U.S. Nat. Mus., 58, 577-588.

Railliet A. (1896): Sur quelques parasites du dromadaire. C.R. Soc. biol., 10, 489-492.

Ransom B.H. (1907): Notes on parasitic Nematodes including descriptions of new genera and species and observations on life histories. Bull. U.S. Dept. Agric. a. Anim. Indust. Circ., 116, $52 \mathrm{p}$.

Rose J.H. (1959): Nematodirus helvetianus, an Intestinal Worm of Cattle. Veterinary Record, 71, 470-472.

Rudolphi C.A. (1802): Fortsetzung der Beobachtungen über die Eingeweidewürmer. Arch. Zool. Zoot., 2, 23-25.

Schulz R.S. (1931): Parasitical worms of rabbits and hares and the diseases they cause. State Agricultural Publishing House, Moscou, 238 p.

Tucker H. (1942): Nematodirus tortuosa n. sp. (Nematode) from the rat, Neotoma. J. Parasitol., 28, 159-163.

Yorke W., Maplestone P.A. (1926): The Nematode parasites of vertebrates. I. A. Churchill, édit., London, $536 \mathrm{p}$. 